\title{
Convergent methodologies in cyber-psychology: A case study
}

\author{
DIANE J. SCHIANO \\ Interval Research Corporation, Palo Alto, California
}

\begin{abstract}
"On-line communities" (and especially MUDs-"multiuser domains") are a popular, growing Internet phenomenon. This paper provides an overview of a project designed to provide a careful characterization of what "life" is like in LambdaMOO--a classic social MUD-for most, or at least many, members. A "convergent-methodologies" approach embracing qualitative and quantitative, subjective and objective methods was used to generate a large and rich database on this on-line community in terms of four general categories: (1) users and use, (2) sociality, (3) identity, and (4) spatiality. The evidence thus far appears to debunk some of the more provocative claims of widespread MUD addiction and rampant identity fragmentation on line. While supporting the primary importance of sociality in the MUD, the results also demonstrate the strong prevalence of personal, one-on-one social interactions over larger social gatherings. Finally, some close correspondences between patterns of spatial behavior and spatial cognition "in real life" and in LambdaMOO were found.
\end{abstract}

The use of the Internet to provide a sense of personal connection and community is a large and growing social phenomenon. In particular, MUDs ("multiuser domains") evolved from early text-based adventure games (e.g., "Dungeons and Dragons") to afford more rich and varied forms of on-line behavior and social interaction than is possible through conversational mechanisms (such as email and chat lines) alone. MUDs and MOOs ("MUDs, object-oriented"), though typically still text-based, are shared, persistent, navigable virtual environments in which user-created characters and scriptable objects can interact with one another in surprisingly rich and compelling ways. MUDs now number in the hundreds and have tens of thousands of members worldwide (see Curtis, 1992, for an excellent introduction to MUDding).

A great deal of social science research - and media attention-has focused on MUDs recently, with some prevalent-and often provocative-claims emerging. These include the views that (1) MUD addiction is common, if not widespread (e.g., Rheingold, 1993; Turkle, 1995); (2) the MUD functions primarily as large public "commons" or "great good place" for social interaction (e.g., Rheingold, 1993); (3) identity-and gender-play with multiple characters (or "morphs") is a primary preoccupation of MUDding, perhaps even promoting a "postmodern fragmentation of the psychological sense of self" (e.g., Allen, 1996; Dalaimo, 1995; Turkle, 1994, 1995); and (4) the spatialized nature of MUDs affords a

Contributors to this project include: Ellen Bewersdorff, Herb Colston, Becky Fuson, Josh Loftus, Scott Mainwaring, and Sean White. Special thanks go to Pavel Curtis and to the many members of LambdaMOO, whose generous help made this project possible. Correspondence concerning this article should be sent to $\mathrm{D}$. J. Schiano, Interval Research Corporation, 1801 Page Mill Road, Palo Alto, CA 94304 (e-mail: schiano@interval.com). vivid sense of place or "presence" on line (e.g., Rheingold, 1993).

Research reports in which such claims are made can be richly evocative, theoretically insightful, and deeply critical of traditional psychological research methods (see, e.g., Allen, 1996; Cherny, 1995; Turkle, 1995). However, the generalizations made from this kind of research are difficult to assess, both because they tend to be based solely on qualitative, anecdotal methods - unstructured interviews, case studies, and ethnographic "thick descriptions" - and because of a tendency to use only a small sample of participants, selected without systematic attention to factors affecting representativeness.

The project to be described here was designed to provide a careful characterization of what "life" is like in LambdaMOO - a classic social MUD_for most, or at least many, members. LambdaMOO is one of the oldest, largest, and most frequently reported upon on-line communities in use today. Indeed, most claims made about MUDs in general have been made about LambdaMOO in particular (e.g., Rheingold, 1993; Turkle, 1995). In this project, the primary categories of interest include: (1) users and use, (2) sociality, (3) identity, and (4) spatiality. Our aim was to establish some reliable benchmarks in these areas for the community as a whole, as well as in terms of gender and experience effects. In so doing, we also wanted to provide a reasonable assessment of some of the major popular claims about MUDs.

To achieve these goals, a hybrid, "convergent methodologies" approach, embracing qualitative and quantitative, subjective and objective research methods, was used. This multimethods approach (currently still in progress) is costly in time and effort, but promises to yield a richly informative database in which results can be contextualized and from which robust patterns of findings can be treated with confidence. This paper provides a brief overview of 
the project, its methodologies, and its emerging patterns of results-especially those related to the common claims about MUDs, as discussed above.

\section{METHOD}

\section{Participants}

All participants were active members of LambdaMOO, a purely "social"- as opposed to fantasy/adventure game (like TrekMUSE) or research (like MediaMOO)-MUD (see Cherny, 1995). Created by Pavel Curtis of Xerox PARC, it is one of the largest MUDs extant, with estimates of almost 10,000 members. Access is freely available through a widely publicized telnet address. In over 5 years, the community has developed and changed formal social norms and even civil governance procedures (e.g., Curtis \& Nichols, 1993).

\section{Procedures}

On-line survey. For 1 week, all log-ins to LambdaMOO opened with an invitation to participate in a research survey. Individuals could accept, decline (at any point), or delay participation. Upon acceptance, one entered a "survey room" in which survey questions were presented in an uninterrupted series. Approximately 30 questions were organized by category of interest; responses included numerical estimates, ratings, and open-ended answers. The survey was completed by 550 respondents, $74 \%$ male and $26 \%$ female.

Personal interviews. The survey contained a call for participation by local residents in a follow-up interview conducted by the author. Twelve volunteers ( 8 male and 4 female, most fairly experienced) participated in discussions conducted in an unstructured, conversational manner, with questions aimed at evoking a rich sense of each person in terms of the four categories of interest. Sessions typically lasted $1.5-2 \mathrm{~h}$ and included a map-drawing task.

Time-sampled logs of system data. Public status information on character and room objects for the entire system was logged at approximately 1 -min intervals, $24 \mathrm{~h} /$ day for approximately 2 weeks (twice). This procedure allowed precise characterization of "who was where when," including time logged on, idle time, user firstconnect date (a measure of experience), character morph count, and "presenting gender." Data on over 4,000 users - approximately 6,000 characters (excluding guests), 55\% "male-presenting," $34 \%$ "female-presenting," $10 \%$ "other" (i.e., the character was not classifiable as male or female) - were taken. No data identifying individual users or the content of interactions were recorded.

Further procedures. Additional procedures included: (1) subject-observer descriptive reports; (2) close tracking of the experiences (and-with permission-some content logs) of 2 naive volunteers from initial log-on through several months of participation; (3) a survey of students at a local campus, including some questions designed to be directly comparable to the LambdaMOO survey; and (4) a map-drawing task replication, with responses sent in by postal mail.

\section{Ethical Considerations}

LambdaMOO is a virtual community with real members. Ethical guidelines should be followed in any research, but special care is needed in studying on-line behavior, since the technology affords precise recording of so much of what would otherwise be inaccessible. With direct support of the LambdaMOO system administrator, we adapted and applied APA guidelines (American Psychological Association, 1992) to ensure voluntary informed consent and confidentiality of both user and character identities of all volunteers. The explicit right to reexamine and delete any recorded content was provided. For the time-sampled logs, we respected privacy by using only public data and not recording data identifying individuals or the content of their interactions. System-wide and mailing-list messages and character-based notices informed MUD members of our research intent.

\section{RESULTS AND DISCUSSION}

Informative patterns of initial results have begun to emerge from this project. These are briefly characterized below.

\section{Users and Use}

Survey information and participant observations suggest that despite large individual differences, most LambdaMOOers are fairly young $(M \approx 19$ years), male (perhaps $70 \%-75 \%$ ) and fairly new (logged on less than 6 months). These data converge well with previous reports (e.g., Curtis, 1992) and with growing access through media publicity.

Clear evidence regarding addiction is given in the time-stamped logs. Figure 1 shows time on line in terms of the mean number of hours/day spent on LambdaMOO, by both user experience level and "modal presenting gender" (MPG-i.e., presenting gender of the most commonly used character for each user). Overall, users spent a mean of $1.13 \mathrm{~h} /$ day $(S D=2.37)$, or under $8 \mathrm{~h} /$ week, on the MUD. Note that this is an extremely liberal estimate of MUDding activity, since it does not take into account idle times, which can be extensive. Even with this liberal measure, however, less than $5 \%$ of the users were on line for over $20 \mathrm{~h} /$ week, despite highly publicized reports suggesting MUDding for up to $80 \mathrm{~h} /$ week or more as perhaps not uncommon (e.g., Turkle, 1995). A reliable increase with experience $[F(3,3110)=7.66, p<.05]$ and a trend toward an experience $\times$ MPG interaction $[F(6,3110)=1.90, p<.05]$ were also found in these data. Thus, while some individuals may well use LambdaMOO addictively, the evidence clearly shows that addiction is not widespread in the population.

\section{Sociality}

LambdaMOO affords a wide variety of activities on line. In one survey query, respondents estimated "percent of time spent in: (1) social interaction, (2) exploring the world, (3) building a description, object, or space, (4) gaming and (5) 'other' activities (please describe)." Overall, social interaction accounted for more than half of total time on average ( $M=57.27 \%, S D=30.25)$, with no other activity listed within 40 percentage points. (Effects of gender and rated experience level were found; females and experienced MUDders reported socializing more). Interview reports lend support to this finding; sociality was generally cited as the primary reason for MUDding, and LambdaMOO's peculiar ability to afford a wide range of creative yet constrained forms of social expression and interaction was widely appreciated.

Interview and time-sampled log data suggest that most socializing is done in pairs or small groups, often privately. Figure 2 shows the mean number of active companions (users in the same "room") per user in LambdaMOO, by experience level and modal presenting gender. Mean number of companions overall was only $1.48(S D=1.49)$, and a reliable decrease with experience $[F(3,31098)=$ 


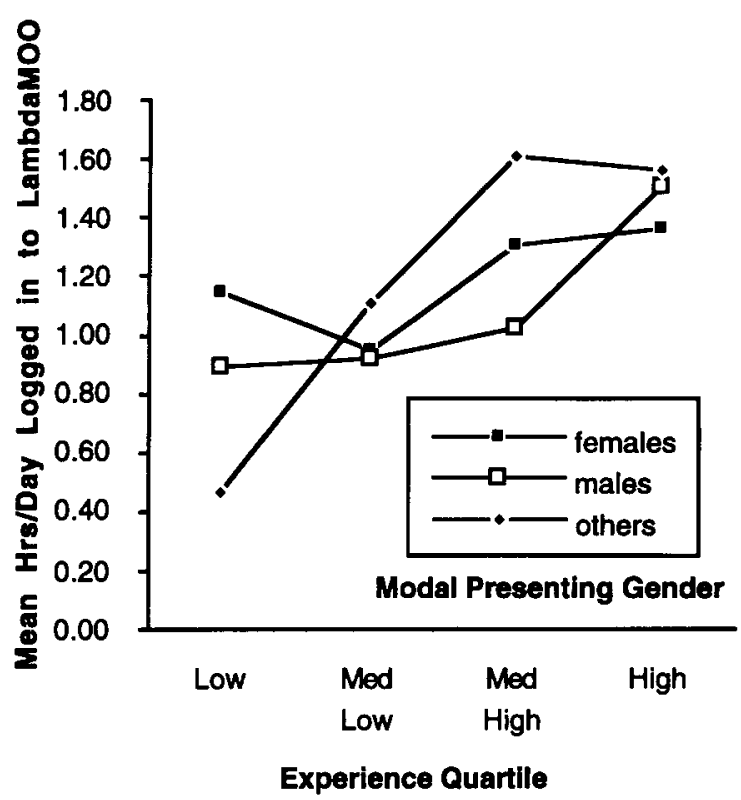

Figure 1. Mean hours/day logged in to LambdaMOO (unadjusted for idle time) by user experience level and modal presenting gender in 2 weeks of time-sampled data.

$10.16, p<.05]$ was found. Thus, while sociality seems to play a primary role in "life in LambdaMOO," these results suggest that it may be typically expressed in a more personal form than characterizations of the MUD as a commons or "great good place" suggest.

\section{Identity}

Sherry Turkle (1994) claims that "you are who you pretend to be" in the MUD, and that "there is no unitary voice ... the self is ... multiplied without limit." Although we had no direct access to the content of character identity in this project, we did find that most people report - in both surveys and interviews - using only one primary character. Interview results further suggest that primary characters are often slightly idealized or fanciful versions of one's view of oneself, with morphs serving more as special-purpose tools or "costumes" than true alternative identities. Indeed, a growing awareness of social pressures to maintain the accountability afforded by a single identity was reported. As one person put it, "pseudonymity is not anonymity." Most people also report speaking in their "own voice" most of the time, regardless of character. Survey respondents rated frequency of "role-playing" a character rather than "being yourself"; the results were moderate to low $(M=2.70$, $S D=1.61$ on a 7 -point scale).

The time-sampled logs show that less than $25 \%$ of players $(M=22.45, S D=41.75)$ ever morphed identity - changed character - within the 2-week observation period; of these, fewer than half ever changed gender. These data are shown in Figure 3, by experience level and modal presenting gender. Users who did morph used a total of three characters $(M=3.05, S D=1.92)$ on the average, but the vast majority of their time $(M=81.44 \%$,
$S D=17.7)$ was spent in a single "main character." Figure 4 presents percentage of time spent in main character by user experience level and modal presenting gender. Significant effects of experience $[F(3,689)=2.82$, $p<.05]$ and an experience $\times$ MPG interaction $[F(6,689)$ $=2.77, p<.05]$ were also found in these data. Taken together, the results thus far suggest that although explorations of multiple identities may be a major activity for some LambdaMOOers, it is not a primary preoccupation for most.

\section{Spatiality}

A special focus of this project centered on spatial behavior and spatial cognition in the MUD; a great deal of additional data were collected on this topic (see also Schiano, 1996). Many of the results, especially those involving complex or multiple-coding techniques - as for the map drawings and open-ended survey responses (e.g., describing navigation strategies or testing spatial memory) - require more complete description and qualification than is possible here; only a brief overview can be provided. In short, initial findings suggest some correspondences between patterns of spatial behavior and spatial cognition "in real life" and in LambdaMOO, even to the extent of replicating some classic gender and experience effects. For example, males report exploring and navigating the space more than females (some support for this is suggested in the time-sampled logs); they are also more likely to report using "mental maps" and customized navigation aids.

In addition, patterns of spatial memory errors (e.g., left-right vs. forward-back reversals) and recall of spatial and "visual" detail gleaned from interactions with the text-based LambdaMOO environment appear similar to those gained from interactions with the physical environ-

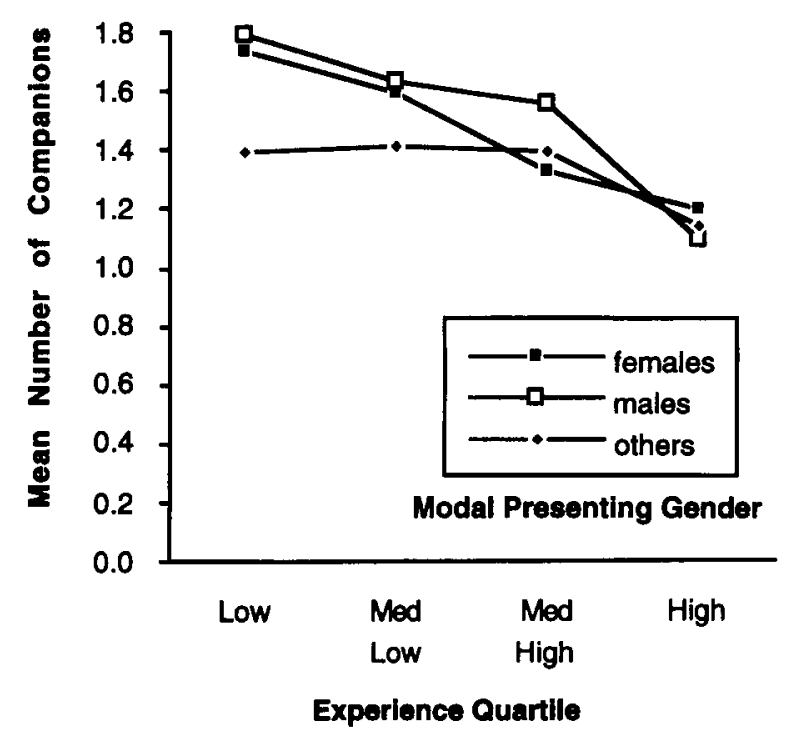

Figure 2. Mean number of companions per user in LambdaMOO (unadjusted for idle users) by user experience level and modal presenting gender in 2 weeks of time-sampled data. 


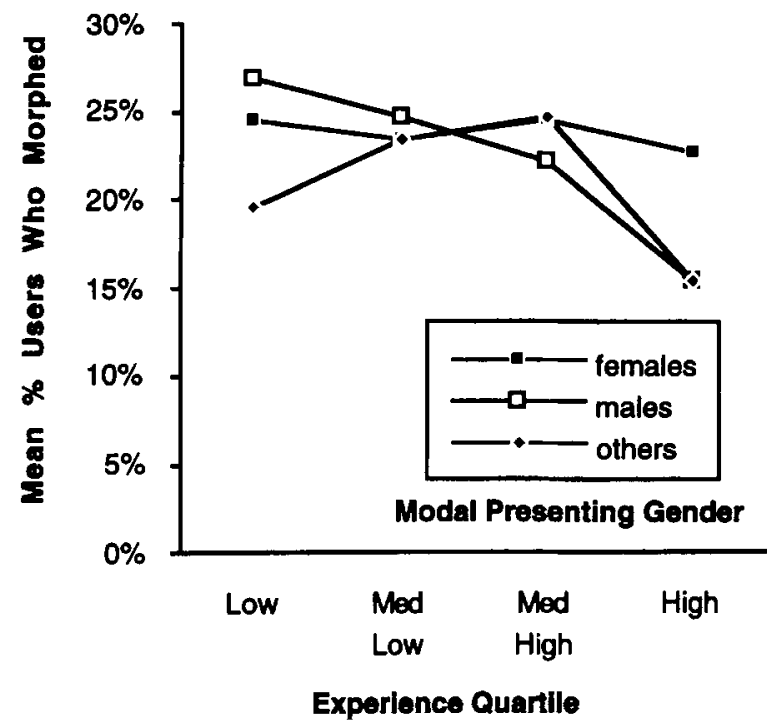

Figure 3. Mean percentage of users who morphed (changed) character by user experience level and modal presenting gender in $\mathbf{2}$ weeks of time-sampled data.

ment - including responses to comparable survey questions from students on a local university campus. This pattern of findings may suggest that the spatialized LambdaMOO environment, though text-based, does provide a sense of place or "presence" for many members. When completed, the corpus of results, including analyses of map drawings, logged "movement" through the MUD, and responses to a series of directly comparable survey questions regarding the visual and spatial characteristics of LambdaMOO and the Stanford campus environments,

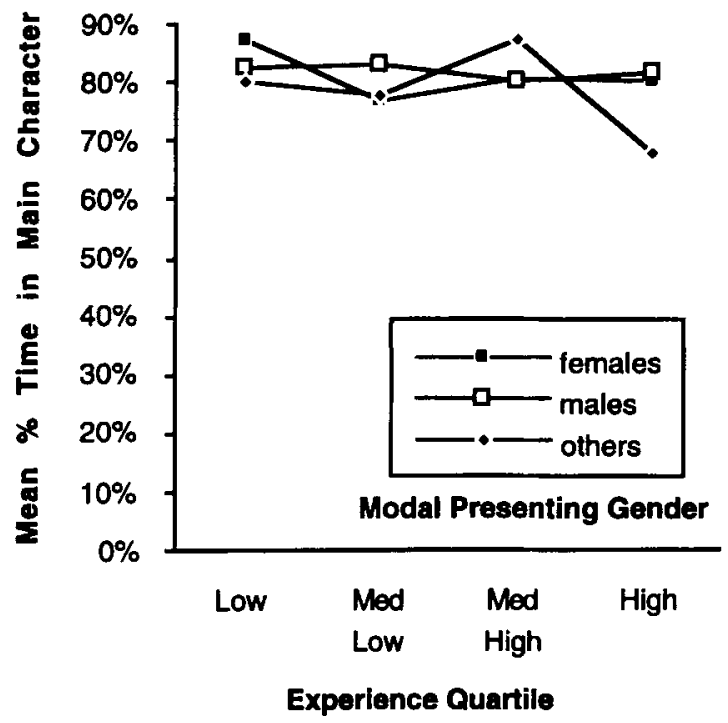

Figure 4. Mean percentage of time spent in main character by user experience level and modal presenting gender in 2 weeks of time-sampled data. could aid our understanding of both basic and applied issues in the field of spatial cognition.

\section{Summary and Conclusions}

Thus far, the "convergent methodologies" approach appears to be fulfilling its promise, yielding a large and rich database from which robust patterns of convergent results can be reported. These data have helped us to begin to characterize carefully a classic on-line community in terms of four broad categories of interest: (1) users and use, (2) sociality, (3) identity, and (4) spatiality. The evidence thus far appears to debunk some of the more provocative claims of widespread MUD addiction and rampant identity fragmentation on line. While supporting the primary importance of sociality in the MUD, the results also demonstrate the strong prevalence of personal, one-on-one social interactions over larger social gatherings. Finally, some correspondences between patterns of spatial behavior and spatial cognition "in real life" and in LambdaMOO were found.

In some sense, this project serves as a "case study" of a research program in which a synergy of qualitative and quantitative, subjective and objective methodologies was achieved. It is our conviction that robust patterns of convergent results derived from such careful recording and reflecting upon on-line behavior can be accepted with confidence. Moreover, we predict that contextualized observations of on-line behavior will come to be seen as extremely useful tools for modeling behavior "in real life" as well as vice versa. We hope that this project will serve to provide some useful first steps - in both directions.

\section{REFERENCES}

Allen, C. (1996). Virtual identities: The social construction of cyberselves. Unpublished doctoral dissertation, Northwestern University. american Psychological Association (1992). Ethical principles of psychologists and code of conduct. American Psychologist, 47, 1597 1611.

CHERNy, L. (1995). The MUD register: Conversational modes of action in a text-based virtual reality. Unpublished doctoral dissertation, Stanford University.

CurTIS, P., (1992). MUDding: Social phenomenon in text-based virtual realities. Intertrek, 3(3), 26-34.

CURTIS, P. \& Nichols, D. (1993, May). MUDs grow up: Social virtual reality in the real world. Paper presented at the meeting of the Third International Conference on Cyberspace, Austin, TX.

DaLAIMO, D. M. (1995). The simulation of selfhood in cyberspace. Unpublished doctoral dissertation, University of Nevada, Las Vegas.

RHEINGOLD, H. (1993). The virtual community: Homesteading on the electronic frontier. New York: Addison-Wesley.

SCHIANo, D. J. (1996, November). Mental models of "MUDs": Just like being... where?" Paper presented at the meeting of the Psychonomic Society, Chicago.

TURKLE, S. (1994). Constructions and reconstructions of self in virtual reality: Playing in MUDs. Mind, Culture \& Activity, 1(3), 158-167.

TURKLE, S. (1995). Life on the screen: Identity in the age of the Internet. New York: Simon \& Schuster.

(Manuscript received October 1, 1996; revision accepted for publication December 19, 1996.) 